\title{
Identification and Protective Efficacy of Eimeria tenella Rhoptry Kinase Family Protein 17
}

\author{
Xiaoxin Liu ${ }^{1,+}$, Bingjin Mu ${ }^{1,+}$, Wenbin Zheng ${ }^{1}$, Yijing Meng ${ }^{1}$, Linmei Yu ${ }^{1}$, Wenwei Gao ${ }^{1}$, Xingquan Zhu ${ }^{1,2}$ \\ and Qing Liu $1, *$ (1)
}

1 College of Veterinary Medicine, Shanxi Agricultural University, Jinzhong 030801, China; vetlxx@163.com (X.L.); mbj142301@163.com (B.M.); wenbinzheng1@126.com (W.Z.); mmmmeng_y@163.com (Y.M.); ylm0719@163.com (L.Y.); sxndgaowenwei@163.com (W.G.); xingquanzhu1@hotmail.com (X.Z.)

2 Key Laboratory of Veterinary Public Health of Higher Education of Yunnan Province, College of Veterinary Medicine, Yunnan Agricultural University, Kunming 650201, China

* Correspondence: lqsxau@163.com

+ These authors contributed equally to this work.

check for updates

Citation: Liu, X.; Mu, B.; Zheng, W.; Meng, Y.; Yu, L.; Gao, W.; Zhu, X.; Liu, Q. Identification and Protective Efficacy of Eimeria tenella Rhoptry Kinase Family Protein 17. Animals 2022, 12, 556. https://doi.org/ $10.3390 /$ ani12050556

Academic Editor: Theo De Waal

Received: 28 December 2021

Accepted: 18 February 2022

Published: 23 February 2022

Publisher's Note: MDPI stays neutral with regard to jurisdictional claims in published maps and institutional affiliations.

Copyright: (C) 2022 by the authors. Licensee MDPI, Basel, Switzerland. This article is an open access article distributed under the terms and conditions of the Creative Commons Attribution (CC BY) license (https:// creativecommons.org/licenses/by/ $4.0 /)$.
Simple Summary: Approximately 8000 genes of Eimeria tenella have been found by genome sequencing, whereas very few data are currently available regarding E. tenella rhoptry kinase family proteins. In this study, the coding sequence of the rhoptry kinase family protein 17 of E. tenella (EtROP17) was cloned and expressed in Escherichia coli, and then the protective efficacy of the recombinant EtROP17 (rEtROP17) was assessed in chickens. Sequence analysis showed that a single base difference at position 1901 of the ROP17 of the SD-01 strain was observed compared with that of the Houghton strain. EtROP17 was expressed in the merozoite stage of E. tenella and may be a potential vaccine candidate against coccidiosis.

\begin{abstract}
Eimeria tenella encodes a genome of approximately 8000 genes. To date, however, very few data are available regarding E. tenella rhoptry kinase family proteins. In the present study, the gene fragment encoding the mature peptide of the rhoptry kinase family protein 17 of E. tenella (EtROP17) was amplified by PCR and expressed in E. coli. Then, we generated polyclonal antibodies that recognize EtROP17 and investigated the expression of EtROP17 in the merozoite stage of E. tenella by immunofluorescent staining and Western blot analysis. Meanwhile, the protective efficacy of rEtROP17 against E. tenella was evaluated in chickens. Sequencing analysis showed that a single base difference at sequence position 1901 was observed between the SD-01 strain and the Houghton strain. EtROP17 was expressed in the merozoite stage of E. tenella. The results of the animal challenge experiments demonstrated that vaccination with rEtROP17 significantly reduced cecal lesions and oocyst outputs compared with the challenged control group. Our findings indicate that EtROP17 could serve as a potential candidate for developing a new vaccine against E. tenella.
\end{abstract}

Keywords: Eimeria tenella; rhoptry kinase family protein 17; merozoite; protective efficacy

\section{Introduction}

Coccidiosis, which is caused by several intracellular intestinal parasites of the genus Eimeria, is the most severe parasitic disease responsible for substantial economic losses to the poultry industry all around the world [1-3]. Annual global economic losses due to coccidiosis are estimated to be USD 3 billion [4]. Conventional strategies used to control the disease depend heavily on chemoprophylaxis $[5,6]$. Meanwhile, live anticoccidial vaccines are used commercially for the control of avian coccidiosis [6]. However, the use of live anticoccidial vaccines or anti-parasitic drugs has disadvantages, such as the occurrence of drug resistance, complex production processes, and potential pathogenicity $[7,8]$. Hence, the 
development of novel control measures such as subunit vaccines has attracted widespread attention [9].

Eimeria tenella is an important species owing to its association with many coccidiosis outbreaks [10]. Approximately 8000 genes of this parasite have been identified by genome sequencing [11]. To date, however, the expression of several proteins, such as rhoptry kinase family proteins, in different developmental phases of the parasite is unknown [12]. E. tenella rhoptry kinase family protein 17 (EtROP17) has been identified in the rhoptry proteome of E. tenella sporozoites, whereas its expression in other developmental phases of the parasite remains unknown [13].

In recent years, a number of $E$. tenella antigens have been tested as candidate antigens for subunit or DNA vaccines, such as surface antigen 16 (SAG16), SAG22, gametocyte 22 antigen, and silent information regulator $2[9,14,15]$. Currently, the protective efficacy of several proteins has been not evaluated, such as EtROP17.

In the present study, the recombinant protein EtROP17 (rEtROP17) was expressed in a prokaryotic expression system. Then, we generated polyclonal antibodies that recognized EtROP17 and investigated the expression of EtROP17 in the merozoite phase of E. tenella. Furthermore, its protective efficacy against $E$. tenella was determined in a chicken challenge model.

\section{Materials and Methods}

\subsection{Parasites and Animals}

Sporulated oocysts of the SD-01 strain of E. tenella were stored in $2.5 \%$ potassium dichromate and propagated in Eimeria-free chicks as previously described [16]. One-dayold Hy-Line layer chicks were reared in isolators under Eimeria-free conditions. BALB/c female mice aged four to five weeks were purchased from the Animal Center of Shanxi Medical University (Shanxi, China).

\subsection{Amplification and Recombinant Expression of EtROP17}

Total RNA was isolated from the E. tenella sporozoites by using TRI Reagent ${ }^{\circledR}$ (SigmaAldrich, St. Louis, MO, USA), which was reverse-transcribed into cDNA using a PrimeScript ${ }^{\mathrm{TM}}$ 1st Strand cDNA Synthesis Kit (Takara, Dalian, China). The gene fragment encoding the mature peptide of EtROP17 (from aa21 to aa665) (Figure 1A) was amplified from the cDNA by PCR using two gene-specific primers: EtROP17-F (5'-GGATCCGTCGACTGTCATCGGGCC-3') and EtROP17-R (5'-GGGGAATTCCTAAACTGAGTTAGTTTCAGACGAG$\left.3^{\prime}\right)$. To construct the recombinant plasmid, the amplified specific fragment was purified and ligated into the BamHI/EcoRI sites of a pET30a vector (designated pET30a-EtROP17). The recombinant vector was verified by endonuclease digestion and DNA sequencing (Tsingke Biotechnology Co., Ltd., Beijing, China). The obtained DNA sequences were compared using the Basic Local Alignment Search Tool (BLAST) (https:/ /blast.ncbi.nlm.nih.gov/Blast.cgi (accessed on 27 June 2021). rEtROP17 was expressed in Escherichia coli BL21 (DE3) and purified using the His60 Ni Gravity Columns (Takara, Dalian, China).

A

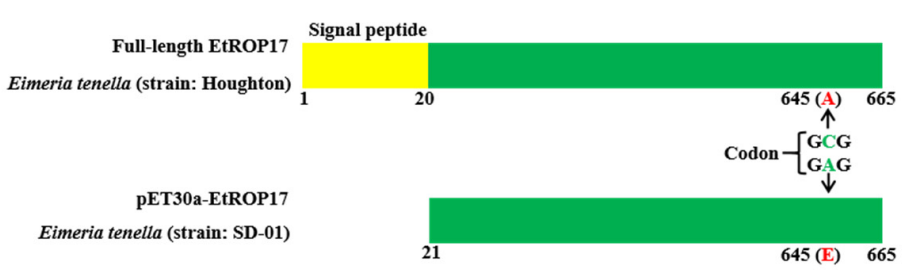

B

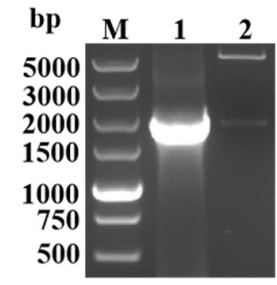

C

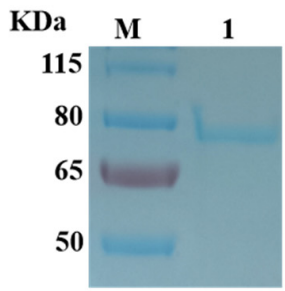

Figure 1. (A) The region of EtROP17 used for expression: the $\mathrm{C}$ to $\mathrm{A}$ transition at nucleotide 1901 converted the amino acid alanine (position 645) to amino acid glutamic acid. (B) Lane M: DL5000 DNA marker; lane 1: the gene fragment of EtROP17 amplified from E. tenella cDNA; lane 2: the recombinant plasmid was identified by digestion. (C) Lane 1: SDS-PAGE analysis of purified rEtROP17. 


\subsection{Generation of Antisera}

The immunized serum against $E$. tenella was prepared according to a previous study [17]. Briefly, chickens reared in Eimeria-free conditions until 2 weeks of age were infected orally with $1 \times 10^{4}$ sporulated oocysts of the SD-01 strain per chicken. Three days after the first challenge, four additional inoculations at 3 day intervals were conducted with 5000 sporulated oocysts per chicken. Five weeks after the final challenge, blood samples were collected by heart puncture. Antiserum was obtained by centrifugation and stored at $-80^{\circ} \mathrm{C}$ until use. Serum collected from unchallenged chickens was used as the normal chicken serum.

The purified rEtROP17 was used as an antigen for generating anti-rEtROP17 serum. Mice were immunized intraperitoneally with the purified rEtROP17 protein $(50 \mu \mathrm{g} / \mathrm{mouse})$ emulsified in Freund's complete adjuvant (Sigma-Aldrich, St. Louis, MO, USA). Subsequently, two additional immunizations at 2 week intervals were administered at the same dose with Freund's incomplete adjuvant (Sigma-Aldrich, St. Louis, MO, USA). Ten days after the last immunization, blood samples were collected and used for the generation of anti-rEtROP17 serum.

\subsection{Immunoblot Analysis of rEtROP17 and Native EtROP17 Protein}

Following resolution by $10 \%$ SDS-PAGE, each purified protein sample was transferred to polyvinylidene fluoride (PVDF) membranes (Millipore, Bedford, MA, USA) using eBlot ${ }^{\mathrm{TM}}$ L1 (GenScript, Nanjing, China). The membranes were separately incubated at $37^{\circ} \mathrm{C}$ for $2 \mathrm{~h}$ with mouse anti-His tag monoclonal antibody (CWBIO, Beijing, China), chicken anti-E. tenella serum, and normal chicken serum. After washing with TBST buffer, the membranes were separately incubated at $37^{\circ} \mathrm{C}$ for $1 \mathrm{~h}$ with the corresponding secondary antibodies: peroxidase-conjugated goat anti-mouse IgG or HRP-conjugated goat antichicken IgY (Abcam, Cambridge, UK). The binding of secondary antibodies was revealed by the enhanced HRP-DAB chromogenic substrate kit (TIANGEN, Beijing, China).

Protein extracts of merozoites were obtained by using RIPA lysis buffer (Beyotime, Nantong, China). Western blot analysis was carried out using mouse anti-rEtROP17 serum as the primary antibody. Goat anti-mouse IgG conjugated with horseradish peroxidase (Abcam, Cambridge, UK) was used as a secondary antibody. The bound antibody was detected using protein enhanced chemiluminescent (ECL) reagent (Thermo Scientific, Waltham, MA, USA). The slide stained with the serum before immunization as the primary antibody was used as the control.

\subsection{Immunofluorescence Analysis of Native EtROP17 Expression}

An immunofluorescence technique was used to detect the expression of EtROP17 in merozoites, as previously described [18]. Merozoites were smeared on a glass slide and air-dried before fixation. Each slide was fixed with $2 \%$ paraformaldehyde for $10 \mathrm{~min}$ at room temperature, and then permeabilized with $0.1 \%$ Triton X-100 in PBS for $10 \mathrm{~min}$. Mouse anti-rEtROP17 serum (dilution of 1:250) was used as the primary antibody. The secondary antibody was FITC-conjugated goat anti-mouse IgG (Abcam, Cambridge, UK) (dilution of 1:5000). The samples were visualized with a Nikon fluorescence microscope (Nikon, Tokyo, Japan).

\subsection{Immunization and Challenge Infection}

One-week-old chickens were weighed and randomly placed into 4 groups, each consisting of 12 birds [9]. The experimental design of immunizations and challenges is shown in Table 1 . The experimental group was vaccinated subcutaneously with $50 \mu \mathrm{g}$ or $100 \mu \mathrm{g}$ of purified rEtROP17 emulsified with Montanide ISA 71 adjuvant (3:7). Chickens in the challenged control and unchallenged control groups were injected subcutaneously with the same $\mathrm{B}$ buffer $\left(100 \mathrm{mM} \mathrm{NaH}_{2} \mathrm{PO}_{4}, 10 \mathrm{mM}\right.$ Tris-HCI) emulsified with the same adjuvant. One week after the first vaccination, a booster vaccination was carried out as described above. All chickens, except those in the unchallenged control group, were infected orally 
with 10,000 freshly sporulated oocysts of the SD-01 strain. The unchallenged control group was given PBS orally.

Table 1. Experimental design of immunizations and challenges.

\begin{tabular}{ccccc}
\hline Groups & Delivery Route & At 7 Days of Age & At 14 Days of Age & Challenge \\
\hline Unchallenged control & Subcutaneous injection & B buffer + ISA 71 & B buffer + ISA 71 & Unchallenged \\
Challenged control & Subcutaneous injection & B buffer + ISA 71 & B buffer + ISA 71 & Challenged \\
rEtROP17-50 $\mu \mathrm{g}$ & Subcutaneous injection & $50 \mu \mathrm{g} \mathrm{rEtROP17+} \mathrm{ISA} \mathrm{71}$ & $50 \mu \mathrm{g} \mathrm{rEtROP17+} \mathrm{ISA} \mathrm{71}$ & Challenged \\
rEtROP17-100 $\mu \mathrm{g}$ & Subcutaneous injection & $100 \mu \mathrm{g}$ rEtROP17+ ISA 71 & $100 \mu \mathrm{g}$ rEtROP17+ ISA 71 & Challenged \\
\hline
\end{tabular}

${ }^{1}$ At 21 days of age, chickens, except for those in the unchallenged control group, were challenged orally with $1 \times 10^{4}$ sporulated oocysts of E. tenella.

\subsection{Evaluation of Protective Efficacy}

The response to infection of chickens in each group was assessed on the basis of body weight gain, lesion score, and oocyst shedding [19]. Body weight gain was determined by weighing the chickens on Day 8 post-challenge and subtracting the body weight on Day 0 post-challenge. Intestinal lesions were scored by using the system described by Johnson and Reid [20]. Fecal samples were collected at Day 6-8 post-challenge, and oocysts per gram were counted using the McMaster's counting technique.

\subsection{Determination of Serum Antibody Levels}

Serum levels of anti-EtROP17 antibodies were examined by ELISA as described elsewhere [8]. Briefly, the 96-well plate was coated with purified rEtROP17 and incubated overnight at $4{ }^{\circ} \mathrm{C}$. Following washing thrice with PBST, the plate was blocked with 5\% skimmed milk in PBST. After washing thrice, wells were incubated with serum samples for $2 \mathrm{~h}$ at $37^{\circ} \mathrm{C}$. Bound antibodies were examined by incubation for $60 \mathrm{~min}$ with HRPconjugated goat anti-chicken IgY antibody and 3, 3, 5, 5-tetramethylbenzidine (TMB). The reaction was stopped by using $1 \mathrm{M} \mathrm{H}_{2} \mathrm{SO}_{4}$, followed by measuring the absorbance at $450 \mathrm{~nm}$ with a microplate spectrophotometer.

\subsection{Statistical Analysis}

Statistical analysis was performed by using a one-way ANOVA Duncan test in the SPSS statistical software (SPSS 26, SPSS Inc., Chicago, IL, USA). Differences between groups at $p$-values smaller than 0.05 were considered to be significantly different.

\section{Results}

\subsection{Amplification, Expression, and Purification}

As shown in Figure 1B, the 1938-bp fragment of EtROP17 was successfully amplified. The resultant plasmid was checked and confirmed by restriction enzyme digestion. Sequencing analysis showed a single base difference at sequence position 1901 between the SD-01 strain and the Houghton strain. The $\mathrm{C}$ to A transition converted the amino acid alanine (position 645) to amino acid glutamic acid (Figure 1A). SDS-PAGE analysis showed that EtROP17 was successfully expressed in the prokaryotic expression system. The molecular weight of the fusion protein was estimated to be $78 \mathrm{KDa}$ (Figure 1C), which is consistent with the size calculated on the basis of the presumptive amino acid sequence.

\subsection{Identification and Immunoreactivity of the Recombinant Protein}

The identification of the purified protein was performed by Western blot using mouse anti-His tag antibody and chicken anti-E. tenella serum, respectively. Western blot analysis using mouse anti-His tag antibody as the primary antibody showed a single immunoreactive band with a relative molecular weight of approximately $78 \mathrm{KDa}$, which is consistent with its deduced size (Figure 2A). Meanwhile, the rEtROP17 could react with chicken anti-E. tenella serum (Figure $2 \mathrm{~B}$ ) but showed no reactivity with normal chicken serum (Figure 2C). 

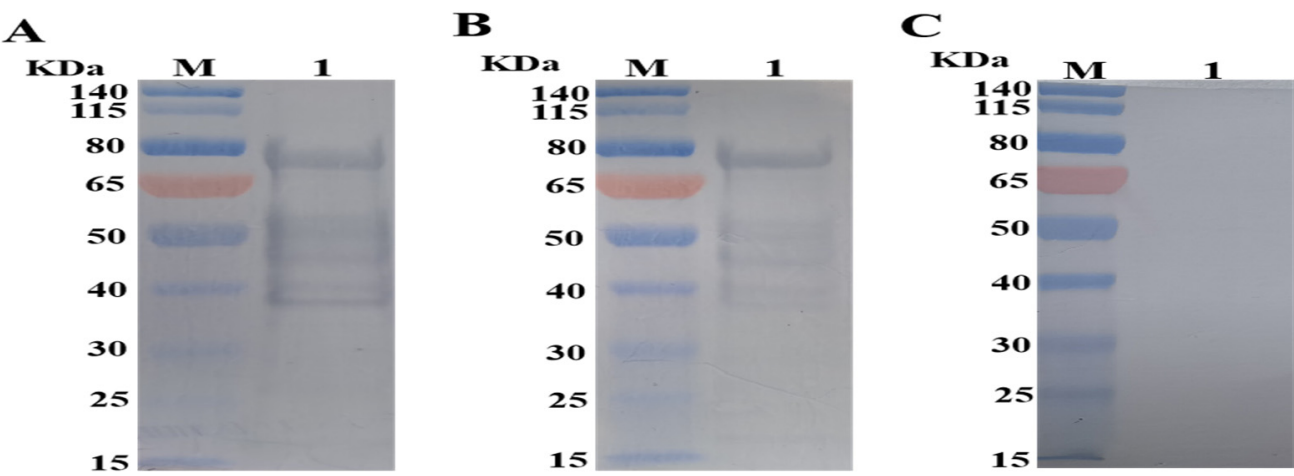

Figure 2. Western blot analysis of rEtROP17 (original Western blot figures are in Figure S1). (A) rEtROP17 was probed with mouse anti-His tag monoclonal antibody. (B) rEtROP17 was probed with chicken anti-E. tenella serum. (C) rEtROP17 was probed with normal chicken serum.

\subsection{Expression Analysis of Native EtROP17 in Merozoites}

The expression of EtROP17 in merozoites of E. tenella was determined using an indirect immunofluorescence technique. Merozoites were clearly labeled by mouse anti-rEtROP17 serum (Figure 3A). Control reactions using normal mouse serum as the primary antibody showed no staining (Figure 3A).

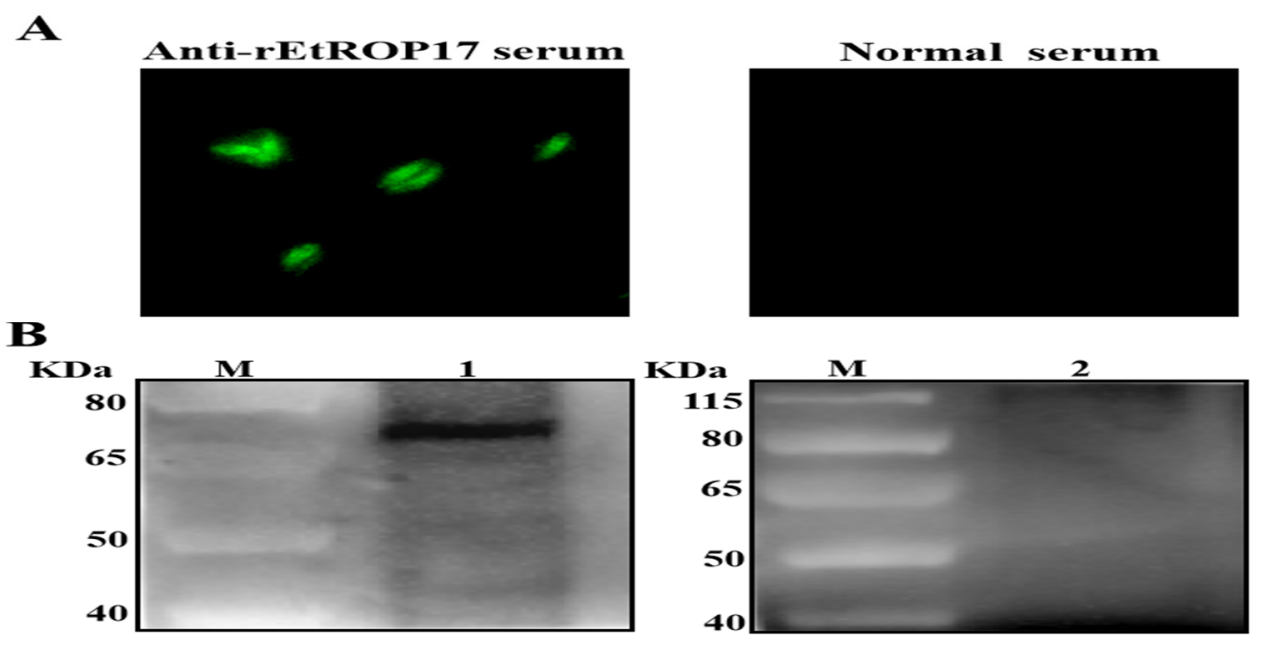

Figure 3. (A) Immunofluorescent staining of E. tenella merozoites, using mouse anti-rEtROP17 serum and normal mouse serum, respectively. (B) Lane M: protein marker; lanes 1-2: Western blot analysis of protein extracts of merozoites using mouse anti-rEtROP17 serum and normal mouse serum, respectively (original Western blot figures are in Figure S2).

The expression of EtROP17 in merozoites was also detected through Western blot. Using mouse anti-rEtROP17 serum as the primary antibody, a unique band of approximately $71 \mathrm{KDa}$ was observed (Figure 3B). The relative molecular weight was consistent with its deduced size. In contrast, no band was observed in the control group (Figure 3B).

\subsection{IgY Titers and Protective Efficacy of Vaccination on E. tenella Challenge}

The IgY titers of vaccinated chickens were significantly higher than those of the challenged control and the unchallenged control groups $(p<0.05)$ (Figure 4A). Resistance to coccidiosis challenge infection was measured by body weight gain, cecal lesion scores, and oocyst output (Table 2). The body weight gain of group rEtROP17-100 $\mu \mathrm{g}$ was the highest, except for the unchallenged control group. However, no significant differences were observed between the immunized groups (50 $\mu \mathrm{g}$ or $100 \mu \mathrm{g}$ of rEtROP17) and the challenged control group, and the body weight gain of group rEtROP17-50 $\mu$ g was similar to that of the challenged control group. (Figure 4B). Cecal lesion scores in chickens immunized 
with $50 \mu \mathrm{g}$ or $100 \mu \mathrm{g}$ of rEtROP17 were significantly lower than those of the challenged control $(p<0.05)$ (Figure 4 C). Chickens vaccinated with rEtROP17 showed a significant increase in oocyst output compared with the challenged control $(p<0.05)$ (Figure 4D).

A
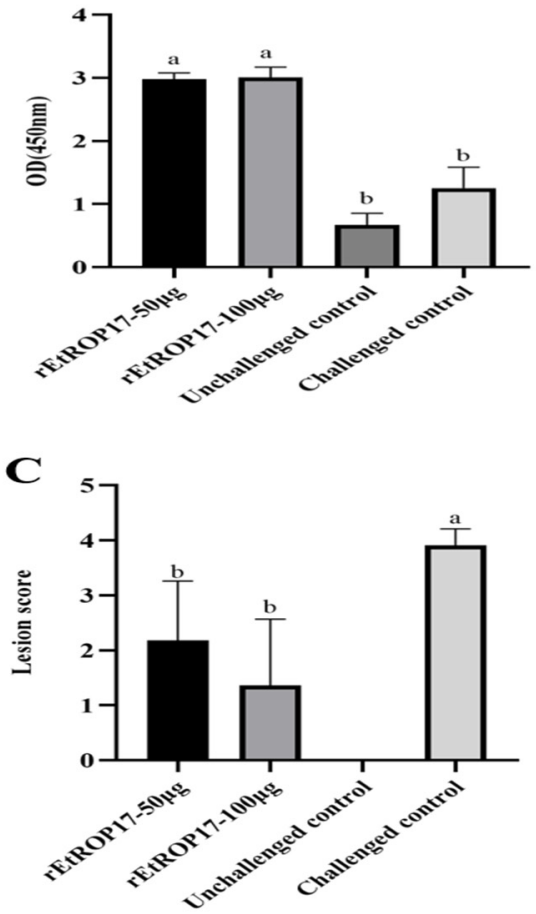
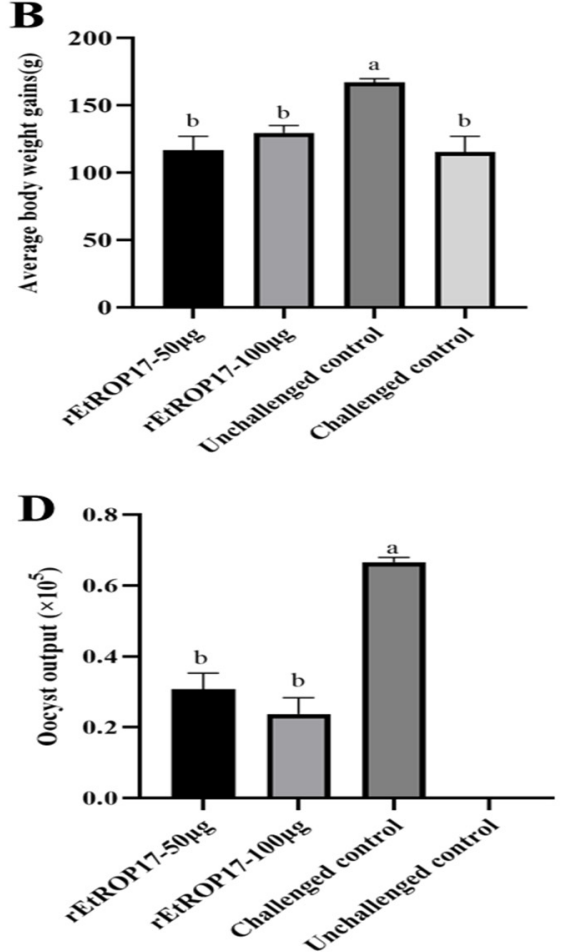

Figure 4. (A) IgY titers. (B) Relative body weight gain. (C) Cecal lesion scores. (D) Oocyst output. Bars sharing the same letters indicate no significant difference.

Table 2. Protective effects in each group.

\begin{tabular}{ccccc}
\hline Groups & $\begin{array}{c}\text { Average Body Weight } \\
\text { Gains (g) }\end{array}$ & $\begin{array}{c}\text { Relative Body Weight } \\
\text { Gain Rate (\%) }\end{array}$ & Lesion Scores & Oocyst Output $\left(\times \mathbf{1 0}^{\mathbf{5}}\right)$ \\
\hline Unchallenged control & $167.32 \pm 9.19^{\mathrm{a}}$ & 100 & $0^{\mathrm{c}}$ & $0^{\mathrm{c}}$ \\
Challenged control & $115.35 \pm 40.89^{\mathrm{b}}$ & 69.95 & $3.91 \pm 0.3^{\mathrm{a}}$ & $0.6665 \pm 0.019^{\mathrm{a}}$ \\
rEtROP17-50 $\mathrm{gg}$ & $116.97 \pm 34.91^{\mathrm{b}}$ & 74.68 & $2.18 \pm 1.08^{\mathrm{b}}$ & $0.3075 \pm 0.064^{\mathrm{b}}$ \\
rEtROP17-100 $\mu \mathrm{g}$ & $129.63 \pm 19.03^{\mathrm{b}}$ & 84.14 & $1.36 \pm 1.21^{\mathrm{b}}$ & $0.238 \pm 0.065^{\mathrm{b}}$ \\
\hline
\end{tabular}

$\mathrm{a}, \mathrm{b}, \mathrm{c}$ : different letters denote statistically significant differences.

\section{Discussion}

In the present study, we cloned and characterized the ROP17 of E. tenella. Sequencing analysis revealed that a single base difference at sequence position 1901 of the ROP17 of the SD-01 strain was observed compared with that of the Houghton strain. The results shown are representative of three independent experiments using high-fidelity DNA polymerase and different cDNA. E. tenella and E. necatrix are considered to be highly pathogenic species [21,22]. Intriguingly, the sequence similarity of ROP17 between E. tenella and E. necatrix is high, whereas the ROP17 of E. tenella, E. maxima, E. acervulina, and E. mitis share low sequence similarity [11]. Hence, ROP17 may represent a virulent factor.

E. tenella has a complex life cycle, and the endogenous stage includes the replication of parasites within chicken intestinal epithelial cells $[23,24]$. The asexual replicative phases are deemed to be responsible for the most damage to intestinal tissues [25]. Previous studies have shown that Toxoplasma gondii ROP17 are vital for survival within host cells [26,27]. The identification of genes expressed in the life cycle of Eimeria species is important for understanding their developmental biology, and the present study primarily aimed to 
determine the expression of EtROP17 in E. tenella merozoites. The expression of EtROP17 in E. tenella merozoites was confirmed by immunofluorescent staining as well as Western blot analysis. To the best our knowledge, this is the first report that EtROP17 is expressed in E. tenella merozoites, which is expected to lay a foundation for further functional studies.

In addition to cell-mediated immunity playing a decisive role in protection against infection, antibodies do exert an effective role in immunity [28]. Western blot analysis suggested that EtROP17 could be recognized by the serum of chickens experimentally infected with E. tenella. This indicated that EtROP17 could be recognized by the chicken immune system and induce an antibody response. This was further confirmed by the subsequent assay in the present study, which showed that chickens immunized with rEtROP17 had a significant increase in IgY titers compared with those of the challenged control and the unchallenged control groups. No significant differences between the challenged and the unchallenged groups in IgY titer were observed, which was consistent with the results of a previous study [29].

Our findings showed that vaccination with rEtROP17 was able to significantly decrease the oocyst output and alleviate cecal lesions as compared to the challenge control group. This demonstrated that EtROP17 might be an effective vaccine candidate against $E$. tenella. However, further studies based on larger numbers of $E$. tenella field isolates from different geographical areas are needed to determine genetic heterogeneity. Meanwhile, it is necessary to evaluate the protective efficacy of EtROP17 showing amino acid changes, as genetic polymorphisms of vaccine candidates may affect protective efficacy [30].

\section{Conclusions}

The present study identified and characterized EtROP17. Sequence analysis showed that a single base difference at position 1901 of the ROP17 of the SD-01 strain was observed compared with that of the Houghton strain. EtROP17 was expressed in the merozoite stage of E. tenella and may serve as a potential candidate for developing vaccines against E. tenella.

Supplementary Materials: The following supporting information can be downloaded at: https: //www.mdpi.com/article/10.3390/ani12050556/s1, Figure S1: Original Western blot figures of Figure 2; Figure S2: Original Western blot figures of Figure 3.

Author Contributions: Q.L., X.Z. and W.G. conceived and designed the study; X.L. and B.M. performed the experiments, analyzed the data, and drafted the manuscript; W.Z., Y.M. and L.Y. participated in the implementation of the study; X.Z., Q.L. and W.Z. critically revised the manuscript. All authors have read and agreed to the published version of the manuscript.

Funding: Project support was provided by the National Natural Science Foundation of China (Grant no. 31902298), the Scientific and Technological Innovation Program of Shanxi Higher Education Institution (Grant no. 2019L0380), the Fund for Shanxi "1331 Project" (Grant no. 20211331-13), the Research Fund for Introduced High-level Leading Talents of Shanxi Province, the Special Research Fund of Shanxi Agricultural University for High-level Talents (Grant no. 2021XG001) and the Yunnan Expert Workstation (Grant no. 202005AF150041).

Institutional Review Board Statement: The study protocol and all experimental procedures have been reviewed and approved by the Animal Ethics Committee of Shanxi Agricultural University (SXAU-EAW-2021C00908; SXAU-EAW-2021M00908).

Informed Consent Statement: Not applicable.

Data Availability Statement: Data supporting reported results will be provided upon request.

Conflicts of Interest: The authors declare no conflict of interest. 


\section{References}

1. Blake, D.P.; Knox, J.; Dehaeck, B.; Huntington, B.; Rathinam, T.; Ravipati, V.; Ayoade, S.; Gilbert, W.; Adebambo, A.O.; Jatau, I.D.; et al. Re-calculating the cost of coccidiosis in chickens. Vet. Res. 2020, 51, 115. [CrossRef] [PubMed]

2. Györke, A.; Kalmár, Z.; Pop, L.; Şuteu, O. The economic impact of infection with Eimeria spp. in broiler farms from Romania. Rev. Bras. Zootec. 2016, 45, 273-280. [CrossRef]

3. Bera, A.; Bhattacharya, D.; Pan, D.; Dhara, A.; Kumar, S.; Das, S. Evaluation of economic losses due to coccidiosis in poultry industry in India. Agric. Econ. Res. Rev. 2010, 23, 91-96.

4. Kadykalo, S.; Roberts, T.; Thompson, M.; Wilson, J.; Lang, M.; Espeisse, O. The value of anticoccidials for sustainable global poultry production. Int. J. Antimicrob. Agents 2018, 51, 304-310. [CrossRef] [PubMed]

5. Dalloul, R.A.; Lillehoj, H.S. Recent advances in immunomodulation and vaccination strategies against coccidiosis. Avian Dis. 2005, 49, 1-8. [CrossRef] [PubMed]

6. Attree, E.; Sanchez-Arsuaga, G.; Jones, M.; Xia, D.; Marugan-Hernandez, V.; Blake, D.; Tomley, F. Controlling the causative agents of coccidiosis in domestic chickens; an eye on the past and considerations for the future. CABI Agric. Biosci. 2021, 2, 37. [CrossRef] [PubMed]

7. Gumina, E.; Hall, J.W.; Vecchi, B.; Hernandez-Velasco, X.; Lumpkins, B.; Mathis, G.; Layton, S. Evaluation of a subunit vaccine candidate (Biotech Vac Cox) against Eimeria spp. in broiler chickens. Poult. Sci. 2021, 100, 101329. [CrossRef] [PubMed]

8. Chen, C.; Tian, D.; Su, J.; Liu, X.; Shah, M.A.A.; Li, X.; Xu, L.; Yan, R.; Song, X. Protective efficacy of rhomboid-like protein 3 as a candidate antigen against Eimeria maxima in chickens. Front. Microbiol. 2021, 12, 614229. [CrossRef] [PubMed]

9. Zhao, H.; Zhu, S.; Zhao, Q.; Huang, B.; Liu, G.; Li, Z.; Wang, L.; Dong, H.; Han, H. Molecular characterization and protective efficacy of a new conserved hypothetical protein of Eimeria tenella. Parasite 2021, 28, 40. [CrossRef] [PubMed]

10. Toulah, F.H.; Esmail, H.A.; Khan, S. The efficacy of Origanum vulgare on Eimeria tenella. J. Egypt. Soc. Parasitol. 2012, 42, 245-250.

11. Reid, A.J.; Blake, D.P.; Ansari, H.R.; Billington, K.; Browne, H.P.; Bryant, J.; Dunn, M.; Hung, S.S.; Kawahara, F.; Miranda-Saavedra, D.; et al. Genomic analysis of the causative agents of coccidiosis in domestic chickens. Genome Res. 2014, 24, 1676-1685. [CrossRef] [PubMed]

12. Diallo, M.A.; Sausset, A.; Gnahoui-David, A.; Silva, A.R.E.; Brionne, A.; Le Vern, Y.; Bussière, F.I.; Tottey, J.; Lacroix-Lamandé, S.; Laurent, F.; et al. Eimeria tenella ROP kinase EtROP1 induces G0/G1 cell cycle arrest and inhibits host cell apoptosis. Cell. Microbiol. 2019, 21, e13027. [CrossRef] [PubMed]

13. Oakes, R.D.; Kurian, D.; Bromley, E.; Ward, C.; Lal, K.; Blake, D.P.; Reid, A.J.; Pain, A.; Sinden, R.E.; Wastling, J.M.; et al. The rhoptry proteome of Eimeria tenella sporozoites. Int. J. Parasitol. 2013, 43, 181-188. [CrossRef] [PubMed]

14. Rafiqi, S.I.; Garg, R.; Ram, H.; Reena, K.K.; Asari, M.; Kumari, P.; Kundave, V.R.; Singh, M.; Banerjee, P.S. Immunoprophylactic evaluation of recombinant gametocyte 22 antigen of Eimeria tenella in broiler chickens. Parasitol. Res. 2019, 118, 945-953. [CrossRef]

15. Dong, H.; Yang, S.; Zhao, Q.; Han, H.; Zhu, S.; Zhu, X.; Li, C.; Wang, Z.; Xia, W.; Men, Q.; et al. Molecular characterization and protective efficacy of silent information regulator 2A from Eimeria tenella. Parasites Vectors 2016, 9, 602. [CrossRef]

16. Fetterer, R.H.; Barfield, R.C. Characterization of a developmentally regulated oocyst protein from Eimeria tenella. J. Parasitol. 2003, 89, 553-564. [CrossRef]

17. Zhang, Z.; Wang, S.; Li, C.; Liu, L. Immunoproteomic analysis of the protein repertoire of unsporulated Eimeria tenella oocysts. Parasite 2017, 24, 48. [CrossRef]

18. Liu, Q.; Chen, Z.; Shi, W.; Sun, H.; Zhang, J.; Li, H.; Xiao, Y.; Wang, F.; Zhao, X. Preparation and initial application of monoclonal antibodies that recognize Eimeria tenella microneme proteins 1 and 2. Parasitol. Res. 2014, 113, 4151-4161. [CrossRef]

19. Zhang, J.; Chen, P.; Sun, H.; Liu, Q.; Wang, L.; Wang, T.; Shi, W.; Li, H.; Xiao, Y.; Wang, P.; et al. Pichia pastoris expressed EtMic2 protein as a potential vaccine against chicken coccidiosis. Vet. Parasitol. 2014, 205, 62-69. [CrossRef]

20. Johnson, J.; Reid, W.M. Anticoccidial drugs: Lesion scoring techniques in battery and floor-pen experiments with chickens. Exp. Parasitol. 1970, 28, 30-36. [CrossRef]

21. Wang, P.; Li, J.; Gong, P.; Wang, W.; Ai, Y.; Zhang, X. An OTU deubiquitinating enzyme in Eimeria tenella interacts with Eimeria tenella virus RDRP. Parasites Vectors 2018, 11, 74. [CrossRef]

22. Su, S.; Hou, Z.; Liu, D.; Jia, C.; Wang, L.; Xu, J.; Tao, J. Comparative transcriptome analysis of Eimeria necatrix third-generation merozoites and gametocytes reveals genes involved in sexual differentiation and gametocyte development. Vet. Parasitol. 2018, 252, 35-46. [CrossRef]

23. Rieux, A.; Gras, S.; Lecaille, F.; Niepceron, A.; Katrib, M.; Smith, N.C.; Lalmanach, G.; Brossier, F. Eimeripain, a cathepsin B-like cysteine protease, expressed throughout sporulation of the apicomplexan parasite Eimeria tenella. PLoS ONE 2012, 7, e31914 [CrossRef]

24. Yan, M.; Cui, X.; Zhao, Q.; Zhu, S.; Huang, B.; Wang, L.; Zhao, H.; Liu, G.; Li, Z.; Han, H.; et al. Molecular characterization and protective efficacy of the microneme 2 protein from Eimeria tenella. Parasite 2018, 25, 60. [CrossRef] [PubMed]

25. Tian, E.J.; Zhou, B.H.; Wang, X.Y.; Zhao, J.; Deng, W.; Wang, H.W. Effect of diclazuril on intestinal morphology and SIgA expression in chicken infected with Eimeria tenella. Parasitol. Res. 2014, 113, 4057-4064. [CrossRef]

26. Guo, M.; Sun, J.; Wang, W.T.; Liu, H.Y.; Liu, Y.H.; Qin, K.R.; Hu, J.R.; Li, X.Y.; Liu, H.L.; Wang, W.; et al. Toxoplasma gondii ROP17 promotes autophagy via the Bcl-2-Beclin 1 pathway. Folia Parasitol 2021, 68, 2021.016. [CrossRef]

27. Li, J.X.; He, J.J.; Elsheikha, H.M.; Chen, D.; Zhai, B.T.; Zhu, X.Q.; Yan, H.K. Toxoplasma gondii ROP17 inhibits the innate immune response of HEK293T cells to promote its survival. Parasitol. Res. 2019, 118, 783-792. [CrossRef] [PubMed] 
28. Constantinoiu, C.C.; Molloy, J.B.; Jorgensen, W.K.; Coleman, G.T. Antibody response against endogenous stages of an attenuated strain of Eimeria tenella. Vet. Parasitol. 2008, 154, 193-204. [CrossRef]

29. Zhao, P.; Wang, C.; Ding, J.; Zhao, C.; Xia, Y.; Hu, Y.; Zhang, L.; Zhou, Y.; Zhao, J.; Fang, R. Evaluation of immunoprotective effects of recombinant protein and DNA vaccine based on Eimeria tenella surface antigen 16 and 22 in vivo. Parasitol. Res. 2021, 120, 1861-1871. [CrossRef] [PubMed]

30. Võ, T.C.; Naw, H.; Flores, R.A.; Lê, H.G.; Kang, J.M.; Yoo, W.G.; Kim, W.H.; Min, W.; Na, B.K. Genetic Diversity of Microneme Protein 2 and Surface Antigen 1 of Eimeria tenella. Genes 2021, 12, 1418. [CrossRef] 